\title{
Impact of cathode evaporation on a free-burning arc
}

\author{
K Etemadi†, G Y Zhaoł and J Mostaghimi§ \\ † State University of New York at Buffalo, Electrical and Computer Engineering, \\ 217A Bonner Hall, Buffalo, NY 14260, USA \\ $\ddagger$ Institute of Mechanics, Chinese Academy of Sciences, Beijing, China \\ $\S$ Chemical Engineering, University of Sherbrooke, Sherbrooke, Quebec, Canada
}

Received 28 February 1989

\begin{abstract}
A free-burning, high-intensity argon arc at atmospheric pressure was modelled during the evaporation of copper from the cathode. The effect of cathode evaporation on the temperature, mass flow, current flow and $\mathrm{Cu}$ concentration was studied for the entire plasma region. The copper evaporates from the tip of the cathode with an evaporation rate of $1 \mathrm{mg} \mathrm{s}^{-1}$. The copper vapour in the cathode region has a velocity of $210 \mathrm{~m} \mathrm{~s}^{-1}$ with a mass concentration of above $90 \%$ within $0.5 \mathrm{~mm}$ from the arc axis. The vapour passes from the cathode toward the anode with a slight diffusion in the argon plasma. Higher temperatures and current densities were calculated in the core of the arc caused by the cathode evaporation.
\end{abstract}

\section{Introduction}

In all plasma arc applications ejection of materials from the electrodes into the plasma causes significant changes in the properties of the discharge. In the tungsten inert gas (TIG) welding process a slight evaporation of materials changes the arc configuration and consequently the heat transfer to the workpiece [1]. As observed in arc furnaces and also in our laboratory, droplets ejected away from the molten anode pool may stick to the high-temperature $(\geqslant 1000 \mathrm{~K})$ zone near the cathode tip where they start to evaporate. The vapour released from the droplet is drawn into the plasma by gas flow caused by the self-magnetic pumping. This creates a concentric vapour beam in the centre of the plasma [2] with a sudden change in the plasma configuration. The configuration remains stable until the droplet is completely evaporated. In switching devices evaporation of electrode materials with low ionisation potential into the centre portion of the arc column enhances the electrical conductivity of the air arc [3]. Here, the knowledge of diffusion of the electrode materials is of particular importance for reducing damage to the switching devices [4] and also to other electric power systems at a fault point.

In the last few years there have been numerous experimental $[3,5]$ and theoretical $[6,7]$ studies to investigate the impact of electrode evaporation on an atmospheric pressure arc. Although the experimental studies provide information about the temperature and species concentration field in the plasma, a better understanding of the impact of the contaminant on the potential field, current density distribution and flow requires more sophisticated diagnostic techniques and/ or the development of a numerical model. In the past, Glickstein [6] developed a one-dimensional model by solving the Elenbaas-Heller equation to study the effects of minor additions of aluminium vapour to a gas mixture of $\mathrm{Ar}$ and $\mathrm{He}$ employed for welding applications. A similar model was also used by other researchers [7] to show the influence of copper vapour originating from the electrodes on the air-arc plasma characteristics.

In the present work, a two-dimensional model has been developed in order to study the impact of copper vapour on an atmospheric pressure free-burning arc as shown in figure 1. The model is an extension of a previous model [8], which includes the species concentration equation in order to account for the diffusion of $\mathrm{Cu}$ in the Ar plasma. Although the actual behaviour of the arc depends largely on the cathode evaporation and simplified assumptions and boundary conditions are adopted, the current model provides considerable insight into the diffusion mechanism of copper vapour on argon plasma.

\section{Modelling of the arc}

A set of conservation equations was solved simultaneously using an iterative, finite difference method [8] for a free-burning argon-copper arc at atmospheric pressure, a current of $150 \mathrm{~A}$, a gap spacing of $1 \mathrm{~cm}$, and a cathode tip of $60^{\circ}$. Thermodynamic and transport 


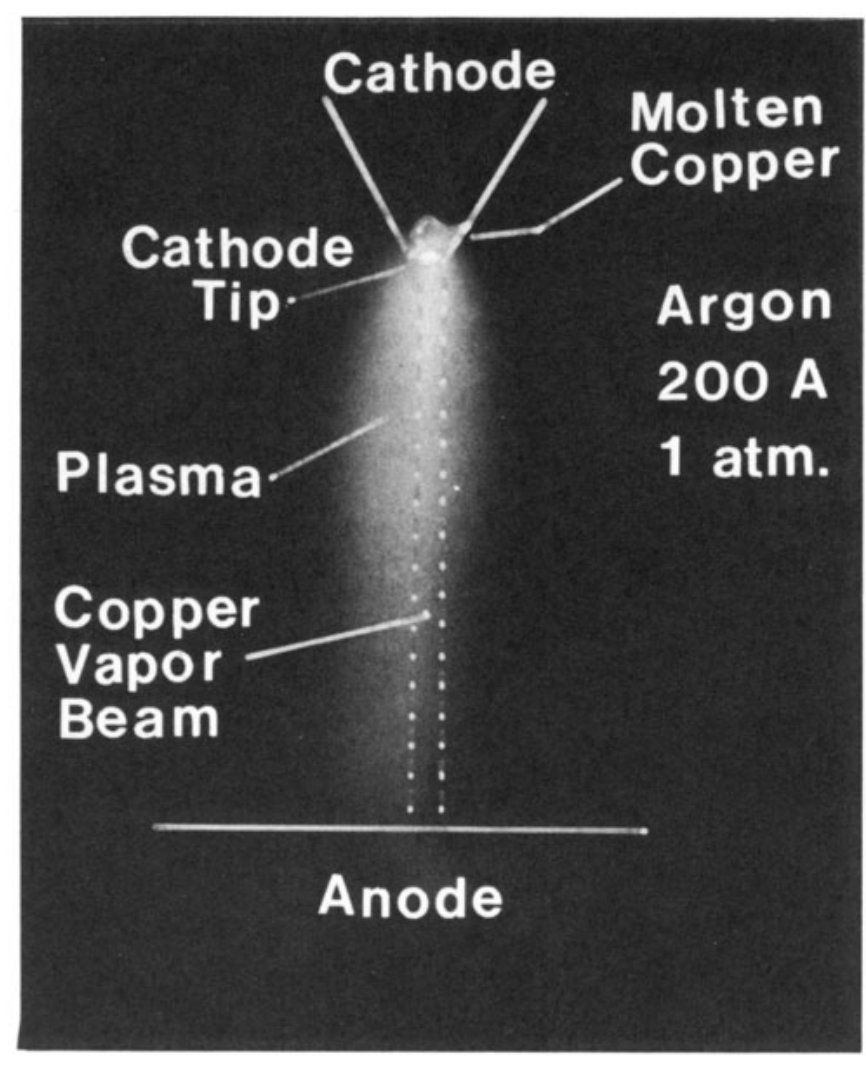

Flgure 1. Photograph of a free-burning arc with copper contamination from cathode.

properties of the argon-copper mixture which are required for the modelling were taken from the report of Dassanayake [9]. The basic assumptions underlying the model are:

(i) The arc is cylindrically symmetric.

(ii) The arc is in the state of local thermodynamic equilibrium.

(iii) The plasma flow is laminar and the arc is in the steady state.

(iv) The plasma is optically thin.

(v) Only the radiation from $\mathrm{Ar}$ is considered in this model.

(vi) Only the diffusion of $\mathrm{Cu}$ and $\mathrm{Ar}$ is considered in this model.

The accuracy of mass, momentum and energy conservation for the entire calculation domain is determined by the ratio of the difference between their inflow and outflow over their inflow into the domain. The values of their accuracy range between $10^{-3}$ and $10^{-5} \%$ and are part of the criteria for convergence.

\subsection{Governing equations}

The governing equations were solved in cylindrical coordinates $(r, x, \theta)$ and are as follows.

Continuity

$$
\frac{\partial}{\partial z}(r \rho u)+\frac{\partial}{\partial r}(r \rho v)=0
$$

$\rho, u$ and $v$ are the mass density, axial and radial velocity components, respectively.

\section{Momentum}

$$
\begin{aligned}
& \rho u \frac{\partial u}{\partial z}+\rho v \frac{\partial u}{\partial r}=-\frac{\partial p}{\partial r}+2 \frac{\partial}{\partial z}\left(\mu \frac{\partial u}{\partial z}\right) \\
& +\frac{1}{r} \frac{\partial}{\partial r}\left(\mu r \frac{\partial u}{\partial r}\right)+\frac{1}{r} \frac{\partial}{\partial r}\left(\mu r \frac{\partial v}{\partial z}\right)+j_{r} B_{\theta} \\
& \rho u \frac{\partial v}{\partial z}+\rho v \frac{\partial v}{\partial r}=-\frac{\partial p}{\partial r}+\frac{\partial}{\partial z}\left(\mu \frac{\partial v}{\partial z}\right)+\frac{2}{r} \frac{\partial}{\partial r}\left(\mu r \frac{\partial v}{\partial r}\right) \\
& +\frac{\partial}{\partial z}\left(\mu \frac{\partial u}{\partial r}\right)-\frac{2 \mu v}{r^{2}}+j_{z} B_{\theta}
\end{aligned}
$$

$p$ is the pressure, $B_{\theta}$ is the self-magnetic field, and $j_{r}$ and $j_{z}$ are the radial and axial current density.

\section{Current continuity}

$$
\frac{\partial j_{z}}{\partial z}+\frac{1}{r} \frac{\partial}{\partial r}\left(j_{r} r\right)=0
$$

or

$$
\frac{\partial}{\partial z}\left(\sigma \frac{\partial \varphi}{\partial z}\right)+\frac{1}{r} \frac{\partial}{\partial r}\left(\sigma r \frac{\partial \varphi}{\partial r}\right)=0
$$

$\sigma$ and $\varphi$ are electrical conductivity and electrical potential, respectively.

$$
\begin{aligned}
& \text { Energy } \\
& \begin{array}{l}
\rho u \frac{\partial h}{\partial z}+\rho v \frac{\partial h}{\partial r}=\frac{\partial}{\partial z}\left(\frac{k}{C_{p}} \frac{\partial h}{\partial z}\right)+\frac{1}{r} \frac{\partial}{\partial r}\left(\frac{k r}{C_{p}} \frac{\partial h}{\partial r}\right) \\
+\frac{j_{z}^{2}+j_{r}^{2}}{\sigma}-S_{r}+\frac{5 k_{\mathrm{B}}}{2 e}\left(\frac{j_{z}}{C_{p}} \frac{\partial h}{\partial z}+\frac{j_{r}}{C_{p}} \frac{\partial h}{\partial r}\right) \\
+\frac{\partial}{\partial z}\left[\sum\left(\rho D-\frac{k}{C_{p}}\right)\left(h_{i}\right) \frac{\partial C_{i}}{\partial z}\right] \\
+\frac{1}{r} \frac{\partial}{\partial r} r\left[\sum\left(\rho D-\frac{k}{C_{p}}\right)\left(h_{i}\right) \frac{\partial C_{i}}{\partial r}\right]
\end{array}
\end{aligned}
$$

$h$ and $h_{i}$ are the enthalpy of the gas mixture and the enthalpy of species $i$ respectively. $C_{p}$ is the specific heat at constant pressure, $k$ is the thermal conductivity, and $k_{\mathrm{B}}$ and $e$ are Boltzmann and elementary charge constants, respectively. $S_{\mathrm{r}}$ is the radiation term [10].

Species conservation

$$
\begin{aligned}
\rho u & \frac{\partial C_{i}}{\partial z}+\rho v \frac{\partial C_{i}}{\partial r} \\
= & \frac{1}{r}\left[\frac{\partial}{\partial z}\left(\rho D r \frac{\partial C_{i}}{\partial z}\right)+\frac{\partial}{\partial r}\left(\rho D r \frac{\partial C_{i}}{\partial r}\right)\right]
\end{aligned}
$$

where

$$
D=\frac{(4 / \sqrt{ } 2)\left(1 / M_{1}+1 / M_{2}\right)^{1 / 2}}{\left[\left(\rho_{1}^{2} \beta_{1}^{2} \mu_{1}^{2} M_{1}\right)^{1 / 4}+\left(\rho_{2}^{2} \beta_{2}^{2} \mu_{2}^{2} M_{2}\right)^{1 / 4}\right]^{2}} .
$$

$C_{i}$ is the mass fraction concentration of species $i . D$ is the binary diffusion coefficient between $\mathrm{Ar}$ and $\mathrm{Cu} . M_{i}$ is the molecular weight of species $i . \rho_{i}$ and $\mu_{i}$ are the 
Table 1. Boundary conditions.

\begin{tabular}{llllllll}
\hline & $\mathrm{AB}$ & $\mathrm{BC}$ & $\mathrm{EC}$ & $\mathrm{EF}$ & $\mathrm{FI}$ & $\mathrm{Al}$ & $\mathrm{DA}$ \\
\hline$u$ & $\partial u / \partial r=0$ & $u=0$ & $\partial u / \partial r=0$ & $\partial \rho u / \partial r=0$ & $u=0$ & $u=C$ \\
$v$ & $v=0$ & $v=0$ & $\partial v / \partial r=C$ & $v=0$ & $v=0$ & $v=0$ \\
$h$ & $\partial h / \partial r=0$ & $h=h_{\text {exp }}$ & $h=h_{0}(1000 \mathrm{~K})$ & $h=h_{0}(1000 \mathrm{~K})$ & $h=h_{1}(3000 \mathrm{~K})$ & $h=h_{\text {exp }}$ & $j=j_{\text {given }}$ \\
$\varphi$ & $\partial \varphi / \partial r=0$ & $\varphi=C$ & $\partial \varphi / \partial r=0$ & & $\partial C_{\mathrm{Ar}} / \partial n=0$ & $C_{\mathrm{Ar}}=0$ & \\
$C_{\mathrm{Ar} r}$ & $\partial C_{\mathrm{Ar} r} / \partial r=0$ & $\partial \mathrm{C}_{\mathrm{Ar}} / \partial z=0$ & $\partial C_{\mathrm{Ar}} / \partial r=0$ & $C_{\mathrm{Ar}}=1$ & & & \\
\hline
\end{tabular}

density and viscosity of the pure compound $i$ at the temperature and total pressure of the mixture, respectively. $\beta_{1}$ and $\beta_{2}$ are constants and their values are equal to 1.385 as given by [11].

Self-induced magnetic field

$$
B_{\theta}=\frac{\mu_{0}}{r} \int_{0}^{r} j_{z} \xi \mathrm{d} \xi
$$

where $\mu_{0}$ is the permeability of the vacuum.

\subsection{Boundary conditions}

The conservation equations were solved with the boundary conditions described in table 1 with reference to the calculation domain shown in figure 2 .

Most of the boundary conditions are the same as described in [12]. While ABCEFA represents the main calculation domain, $A B C D A$ is used for solving the current continuity equation. The Gaussian current density distribution

$$
j(r)=j_{\max } \exp (-a r)
$$

was chosen for the boundary along $\mathrm{AD}$ for all cases. The constants $j_{\max }$ and $a$ were calculated based on the electric current and the size of the cathode attachment. Copper is evaporated uniformly from the cathode boundary AI into the plasma with an evaporation rate of $1 \mathrm{mg} \mathrm{s}^{-1}$. This number is an approximate value determined from the size (measured by a close-up picture of the droplets; see figure 1) and evaporation time of the droplets. The current density distribution along the boundary AD is assumed to be unaffected by the copper vapour ejected from the cathode. This is a reasonable assumption due to the negligible changes in electrical conductivity induced by the presence of copper vapour in argon plasma at temperatures above

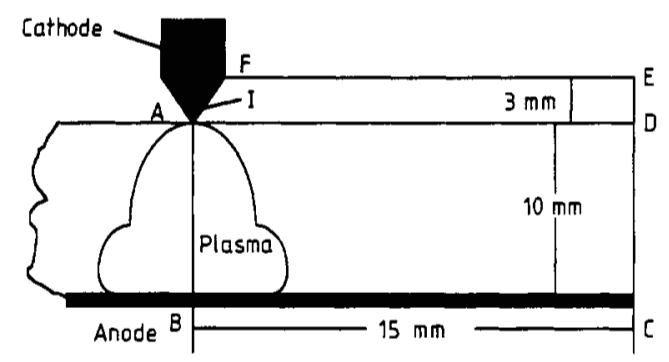

Flgure 2. Arc arrangement and calculation domains.
$14000 \mathrm{~K}$ [9]. The assumptions on boundaries $\mathrm{FE}$ and EC do not have a remarkable impact on the solution for the plasma region. The temperature distribution and flow field within the plasma regions are mainly determined by electromagnetic pumping in the cathode region. Modelling of the entire plasma reactor [13] in which the boundaries are extended to the reactor walls shows major impact only in the lower temperature region of the reactor. The position and temperature distribution of the anode boundary $\mathrm{BC}$ are determined based on the following considerations.

(i) The temperature measurements performed at $0.5 \mathrm{~mm}$ above the anode [12] confirm a sharp temperature drop (as much as $8000 \mathrm{~K}$ in a fraction of a $\mathrm{mm}$ ) immediately above the anode surface meaning isothermal lines below $8000 \mathrm{~K}$ in the anode region of the arc are almost parallel to the anode surface and are concentrated in a very short distance (fraction of a $\mathrm{mm}$ ) above the anode.

(ii) The gas mass flow rate in the region of isotherms below $8000 \mathrm{~K}$ is assumed to be equal to the flow rate on the anode surface with $\rho v=0$ and $\rho u=$ 0 . The position of the anode boundary is therefore shifted from the anode surface to an isothermal line below $8000 \mathrm{~K}$. However, this shift has negligible influence on other plasma regions as confirmed by the present model. In order to choose the most appropriate position for the boundary $\mathrm{BC}$, on one hand, it is desirable to move the anode boundary as close as possible to the anode surface (or to the position of an isothermal line with as close as possible a temperature to the anode surface) to satisfy the velocity boundary conditions. On the other hand, at temperatures below $5000 \mathrm{~K}$ the electrical conductivity of the plasma drops to a negligible value so that electric current cannot flow through the insulating gas to the anode. Consequently, convergence could not be achieved by the model.

For the above-mentioned reason, a temperature of $5000 \mathrm{~K}$ was selected at the anode boundary in the plasma region. Outside the plasma region it is assumed that the temperature drops linearly to the anode temperature $(1000 \mathrm{~K})$ at the anode boundary. The gradients of $u, h, \varphi$ and $C_{\mathrm{Ar}}$ are equal to zero along the centre line $\mathrm{AB}$ due to the symmetry condition.

\section{Results and discussion}

Figure 3 shows the isotherms and the stream function $\left(=\int \rho u r \mathrm{~d} r-\int \rho v r \mathrm{~d} z\right)$ of a $150 \mathrm{~A}$ free-burning arc at 


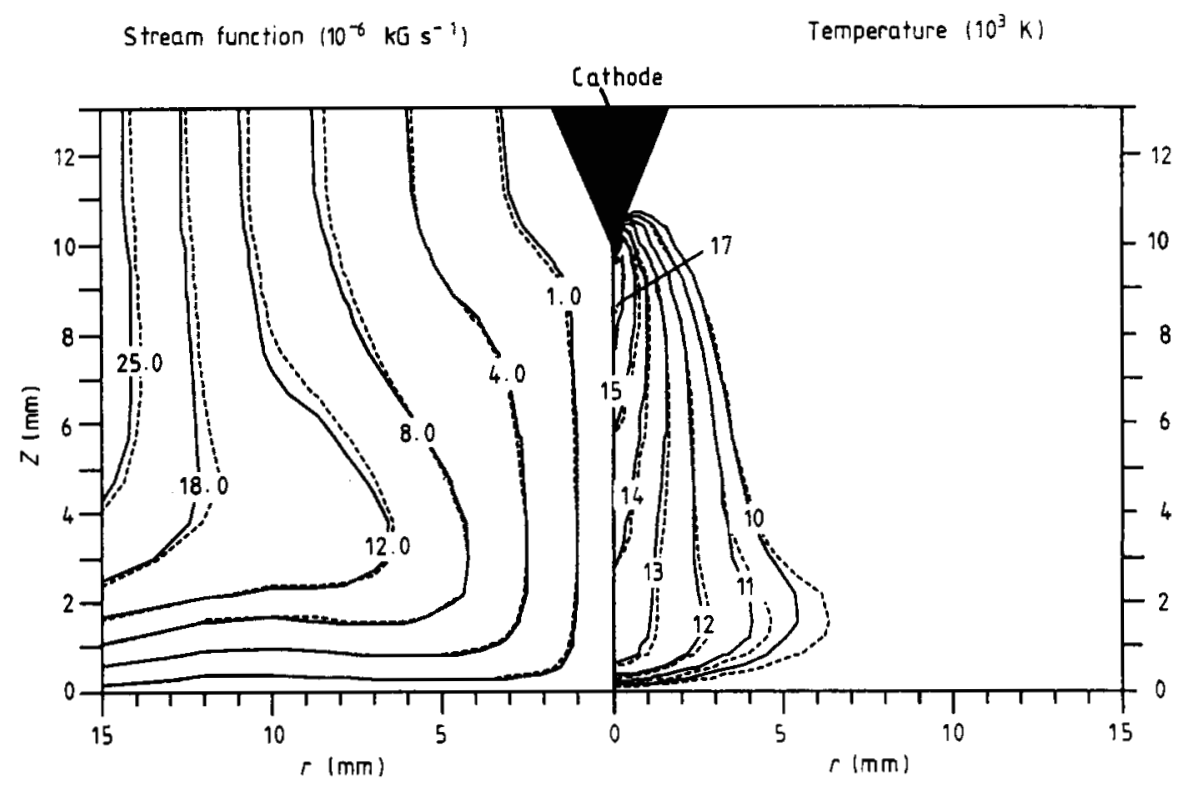

Figure 3. Isotherms and stream function of a free-burning arc. $l=150 \mathrm{~A}, \rho=$ $1 \mathrm{~atm}, m_{\mathrm{b}}=1 \mathrm{mG} \mathrm{s}^{-1}$. Curves: —, with evaporation; - - - without evaporation.

atmospheric pressure and a gap spacing of $1 \mathrm{~cm}$. The full and broken curves represent the results with and without evaporation from the cathode, respectively. In the presence of the copper the temperatures are lower in the fringes and higher in the core of the plasma. The concentration of the copper in the plasma region is shown in figure 4 . The results show that the vapour ejected from the cathode and combined with the argon gas is accelerated toward the anode by the electromagnetic pumping force. Copper vapour remains con-

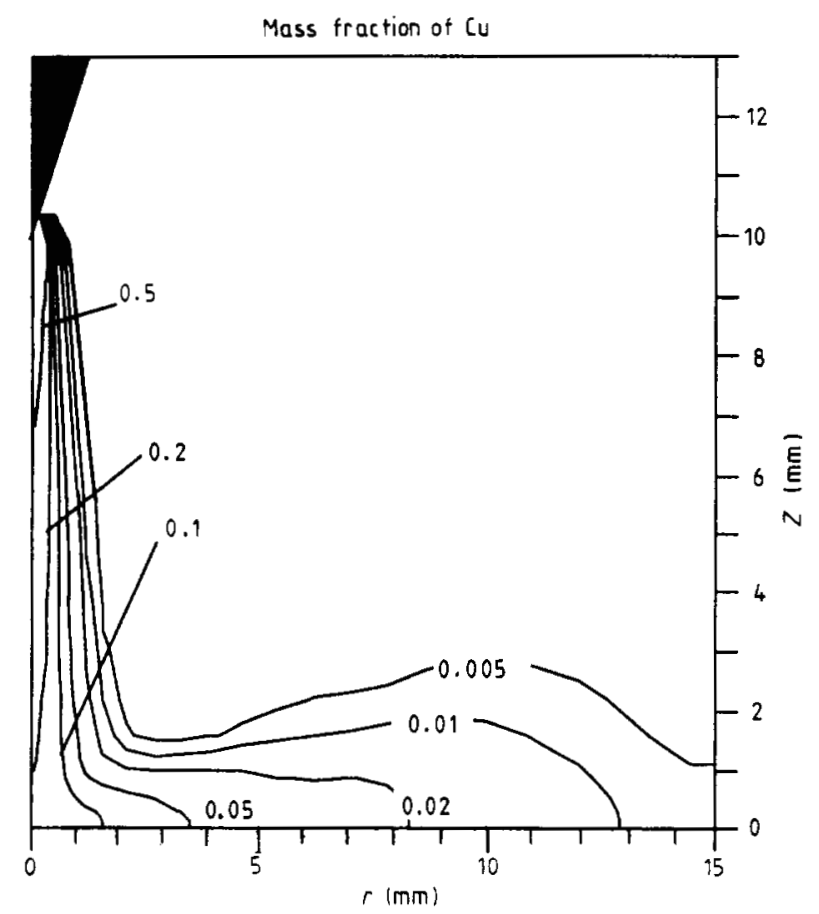

Figure 4. The copper concentration distribution in a $\mathrm{Cu}$ contaminated argon free-burning arc. $m_{\mathrm{t}}=1 \mathrm{mG} \mathrm{s}^{-1}$. centric as it approaches the anode but suddenly spreads in a radial direction on the anode surface. The mass fraction of $\mathrm{Cu}$ is also shown for four different axial locations in figure 5 . The electrical conductivity of the plasma is significantly reduced in the plasma due to the presence of the metallic vapour of $\mathrm{Cu}$ which has lower ionisation potential than Ar. The impact of the $\mathrm{Cu}$ vapour on the electrical current densities is demonstrated in figure 6 . The current flow is more constricted toward the centre of the arc due to the presence of the $\mathrm{Cu}$. The increase in the current density in the core of

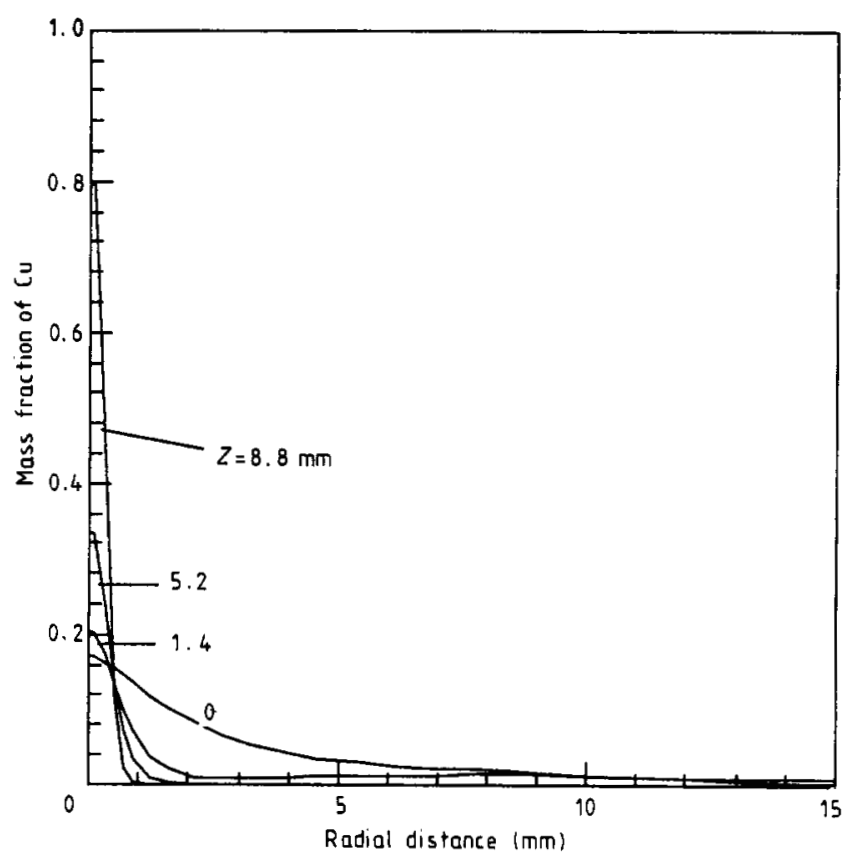

Figure 5. Mass fraction of $\mathrm{Cu}$ at different positions of $Z$. $m_{\mathrm{t}}=1 \mathrm{mG} \mathrm{s}^{-1}$. 


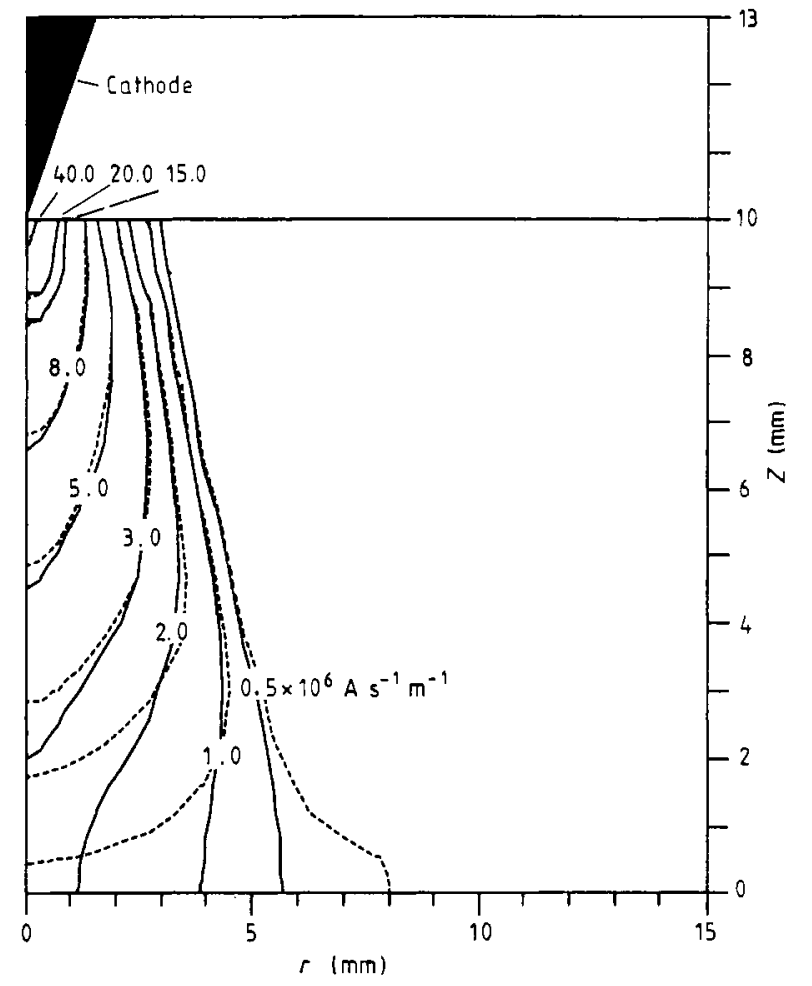

Figure 6. The impact of copper vapour on the electrical current density. $m_{1}=1 \mathrm{mG} \mathrm{s}^{-1}$. Curves: - with evaporation; -- , without evaporation.

the arc is the most significant cause of its temperature increase in the cathode region.

\section{Conclusion}

A free-burning, high-intensity argon arc at atmospheric pressure was modelled during the evaporation of cop- per from the cathode, in order to study the impact of the vapour on the entire plasma region. Copper evaporated from the cathode is drawn toward the anode by self-magnetic pumping and remains concentric between the electrodes as observed experimentally. The electrical conductivity in the entire anode region was increased due to the presence of copper vapour which, in turn, enhanced the constriction of the current toward the centre. Therefore, higher temperatures in the core and lower temperatures in the fringes of the arc were calculated.

\section{References}

[1] Glickstein S S 1976 Welding J. August 222s-9s

[2] Mentel J 1978 Current Interruption in High-Voltage Network (New York: Plenum) pp 119-25

[3] Sakuta T, Kito Y and Miyachi I 1980 IEEE Trans. Plasma Sci. PS-8 29-32

[4] Lins G 1988 IEEE Trans. Plasma Sci. PS-16 433-7

[5] Cheminat B and Andanson P 1985 J. Phys. D: Appl. Phys. 18 2183-92

[6] Glickstein S S 1979 Arc modelling for welding analysis Int. Conf. on Arc Physics and Weld Pool Behaviour (London) 8-10 May 1979 (London: Welding Institute)

[7] Ouajji H, Cheminat B and Andanson P 1987 J. Phys. D: Appl. Phys. 20 635-8

[8] Patankar S V 1980 Numerical Heat Transfer and Fluid Flow (New York: McGraw-Hill)

[9] Dassanayake M and Etemadi K 1989 Bell Aerospace Textron Task \# 7, January

[10] Evans D L and Tankin R D 1967 Phys. Fluids 10 1137-44

[11] Wilke C R $1950 \mathrm{~J}$. Chem. Phys. 14 517-9

[12] Hsu K C, Etemadi K and Pfender E 1983 J. Appl. Phys. 54 1293-310

[13] Etemadi K and Zhao G Y 1989 9th Int. Symp. on Plasma Chemistry (Pugnochiuso, Italy) September 1989 (Bari: IUPAC) 